\title{
Antitumor effects of two newly constructed oncolytic herpes simplex viruses against renal cell carcinoma
}

\author{
XINPING FU ${ }^{1,2,4}$, MIKIHITO NAKAMORI ${ }^{1,2,5}$, LIHUA TAO ${ }^{1,2}$, ROBERT AMATO $^{3}$ and XIAOLIU ZHANG ${ }^{1,2}$ \\ ${ }^{1}$ Department of Pediatrics and ${ }^{2}$ Center for Cell and Gene Therapy, Baylor College of Medicine; ${ }^{3}$ The Methodist Hospital, \\ Houston, TX, USA; ${ }^{4}$ The Second Affiliated Hospital of Chongqing Medical University, Chongqing, P.R. China
}

Received February 1, 2007; Accepted March 12, 2007

\begin{abstract}
Conditionally replicating (oncolytic) herpes simplex viruses (HSVs) have shown clear potential as effective agents for the treatment of solid tumors such as renal cell carcinoma (RCC). To enhance the oncolytic capabilities of first-generation HSVs, we recently developed two new constructs. Synco-2D is derived from HSV-1 and contains two mechanisms to induce cell membrane fusion. FusOn-H2 is derived from HSV-2. It selectively targets the activated Ras signaling pathway in tumor cells and also has the ability to induce cell membrane fusion and apoptosis. We studied the in vitro and in vivo antitumor effects of both Synco-2D and FusOn-H2 against RCC. Both Synco-2D and FusOn-H2 lysed human RCC cells in vitro much more readily than did Baco-1. For in vivo studies, the oncolytic viruses were administered either intratumorally or intravenously to nude mice bearing xenografted human RCC, and the tumor growth rate and animal survival were monitored after treatment. In most instances, the results were compared with those for a first-generation non-fusogenic oncolytic HSV (Baco-1). A single intratumoral injection of either Synco-2D or FusOn-H2 produced a striking effect against xenografted human RCC, in contrast to Baco-1, which produced only moderate antitumor activity. Two intravenous injections of Synco-2D also inhibited the growth of human RCC xenografts, while Baco-1 injections via the same route lacked any measurable antitumor effect. These data demonstrate that the newly constructed oncolytic HSVs have potent activity against established RCC in animal models. Clinical trials to validate these results in cancer patients appear warranted.
\end{abstract}

Correspondence to: Dr Xiaoliu Zhang, Center for Cell and Gene Therapy, Baylor College of Medicine, One Baylor Plaza, Houston, TX 77030, USA

E-mail: xzhang@bcm.tmc.edu

Present address: ${ }^{5}$ Second Department of Surgery, Wakayama Medical University, School of Medicine, Japan

Key words: oncolytic virus, fusogenic, HSV-2, renal cell carcinoma, Ras pathway

\section{Introduction}

Renal cell carcinoma ( $\mathrm{RCC}$ ) is the tenth most common cancer in the US and, in 2004, there were an estimated 35,710 new cases and 12,480 deaths due to this tumor (1). $\mathrm{RCC}$ is both refractory to cytotoxic chemotherapy and radiotherapy (2). Surgical resection (radical nephrectomy) is the cornerstone of curative-intent treatment for localized RCC; however, approximately $30 \%$ of patients with RCC present with metastatic disease, and nephrectomy provides little benefit for such cases (3). In addition, 20-30\% of patients with localized tumors relapse after radical nephrectomy in a median time of 15-18 months. The 5-year survival rate for patients with metastatic RCC generally ranges from 0 to $10 \%$. Recent approval of kinase inhibitors such as sunitinib and sorafenib, which have been shown to reduce the size of metastatic RCC in $26-37 \%$ of patients (4), has improved but not completely altered the bleak outcome of this malignancy. Hence, the exploration of new and more effective treatments for this disease is clearly of great importance.

One experimental therapeutic approach that has shown considerable potential in the treatment of solid tumors such as RCC is the use of conditionally replicating (oncolytic) viruses. These viruses are genetically modified such that they can selectively replicate in tumor cells while sparing normal cells. They have two principal advantages. First, unlike conventional chemotherapy and radiotherapy, they specifically target cancer cells because of their restricted ability to replicate in normal cells (5). Second, as compared with replication-incompetent vectors, they can propagate from initially infected tumor cells to surrounding tumor cells, thereby achieving a large volume of distribution and enhanced antitumor effects. Oncolytic herpes simplex virus (HSV), for example, is commonly constructed by deleting viral genes necessary for efficient replication in normal (non-dividing) cells but not in tumor cells. Such genes, including those encoding thymidine kinase (6), ribonucleotide reductase (7), and uracyl N-glycosylase (8), are necessary to generate sufficient dNTP pools for efficient viral DNA replication in cells where deoxynucleotide pools are low (e.g., quiescent neurons), but are non-essential in tumor cells where deoxynucleotides are abundant (e.g., tumor cells). Thus, viruses modified in this way can preferentially replicate in and kill tumor cells. Another type of oncolytic HSV is derived from mutants with defects in the function of the $\gamma 34.5$ gene, which 
functions as a virulence factor by markedly enhancing the viral burst size of infected cells through suppression of the shutoff of host protein synthesis (9). HSV deleted in $\gamma 34.5$ is severely attenuated for neurovirulence but maintains its ability to replicate in dividing cells (10). Initially designed and constructed for the treatment of brain tumors $(6,11)$, oncolytic HSVs have proved to be effective in treating a variety of other human solid tumors, including renal cell carcinoma (12).

We recently constructed several new oncolytic viruses, with the intent of improving the antitumor potency and specificity. One construct, Synco-2D, derived from type 1 HSV (HSV-1), incorporates two cell-membrane fusion mechanisms (13), enabling it to induce syncytia and thus to kill tumor cells simultaneously by two complementary actions: lytic virus replication and cell membrane fusion. Membrane fusion during syncytia formation may also enhance the spread of Synco-2D among tumor cells. Another construct, FusOn-H2, was derived from type $2 \mathrm{HSV}$ (HSV-2). It was constructed by exploiting a unique feature of the ICPIO gene of HSV-2, which contains in its $\mathrm{N}$-terminus a well-defined region that encodes a serine/threonine protein kinase (PK) activity (14). This PK domain can bind and phosphorylate the GTPase activating protein Ras-GAP, leading to activation of the Ras/MEK/MAPK mitogenic pathway and c-Fos induction and stabilization, and thus is required for efficient HSV-2 replication $(15,16)$. Deletion of the PK domain in FusOn-H2 allows the virus to selectively replicate in (and lyse) human tumor cells with an activated Ras signaling pathway (17). The mutant can also induce syncytia formation in tumor cells, providing an additional oncolytic mechanism that has been shown to enhance its antitumor effect overall (17). As reported here, we evaluated the antitumor effects of these two novel oncolytic HSVs in a series of RCC tumor models and in certain instances, directly compared the results with those produced by first generation oncolytic HSVs.

\section{Materials and methods}

Cell lines and viruses. African green monkey kidney (Vero) cells, and the human RCC lines ACHN and A498 were obtained from American Type Culture Collection (Rockville, MD). All of the cells were cultured in DMEM containing 10\% FBS. Wild-type HSV-2 strain 186 (wt186) (18) was kindly provided by Dr Lawrence Stanberry (University of Texas Medical Branch, Galveston, TX). Both Baco-1 and Synco-2D are derived from HSV-1 and their constructions are described elsewhere $(13,19)$. To construct FusOn-H2, we initially used PCR to amplify the ICP10 left-flanking region of the wild-type HSV-2 strain 186 (wt186) genome (equivalent to nucleotide span 85994-86999 in the HSV-2 genome) and the ribonucleotide reductase domain plus the right-flanking region (equivalent to nucleotide span 88228-89347). These PCR products were cloned together into pNeb193, generating pNeb-ICP10-deltaPK. Hence, the new plasmid contains a mutated ICPIO gene, in which the PK domain (equivalent to nucleotide span 86999-88228) is deleted. Then, the DNA sequence containing the CMV promoter-EGFP gene was PCR amplified from pSZ-EGFP, and the resultant DNA was cloned into the deleted $P K$ locus of pNeb-ICP10-deltaPK, generating pNeb-PKF-2. During the design of PCR amplification strategies, the $E G F P$ gene was fused in-frame with the remaining RR domain of the ICP10 gene, so that the new protein product of this gene contained the intact EGFP. The modified ICPIO gene was inserted into the genome of wt 186 by homologous recombination. The recombinant virus (FusOn-H2) was identified by screening for GFP-positive plaques. Full details of FusOn-H2 construction are published elsewhere (17).

Virus stocks were prepared by infecting Vero cells with 0.01 plaque-forming units (pfu) per cell. Viruses were harvested 2 days later and purified as described (13). The purified viruses were titrated, divided into aliquots and stored at $-80^{\circ} \mathrm{C}$ until use.

In vitro characterization of oncolytic HSVs. For in vitro phenotypic characterization, we infected cells with the viruses at $0.01 \mathrm{pfu}$ per cell and incubated them for $48 \mathrm{~h}$ before photomicrographs were taken. The cytolytic effect of the viruses was measured by infecting cells in 24-well plates with each virus at different multiplicities of infection (MOIs) or by leaving them uninfected. Cell viability was determined by trypan-blue staining. To determine the percentage of cell viability, we divided the number of viable cells from each infected well by the number of cells from the uninfected well. All experiments were performed in triplicate with mean numbers used in the final calculation.

Animal studies. Six-week-old female Hsd athymic (nu/nu) mice were purchased from Harlan (Indianapolis, IN). All experimental procedures involving animals were approved by the Baylor College of Medicine Animal Care and Use Committee. The human RCC ACHN line of RCC was cultured in standard conditions and harvested in log-phase with $0.05 \%$ trypsin-EDTA. The cells were washed twice with serum-free medium before being resuspended in PBS at a concentration of $2 \times 10^{6}$ cells $/ \mathrm{ml}$. For subcutaneous tumor cell implantation, $2 \times 10^{5}$ ACHN cells (in a volume of $100 \mu \mathrm{l}$ ) were injected into the right flanks of nude mice. When tumor diameters reached approximately $5 \mathrm{~mm}$, the animals were given a single intratumor injection of oncolytic HSVs at a dose of $1 \times 10^{7} \mathrm{pfu}$ (Baco-1 or Synco-2D) or 5x106 pfu (FusOn-H2), respectively. Alternatively, mice received two intravenous injections (with a 7-day interval) of either Baco-1 or Synco-2D. For intratumoral injections, viruses in a volume of $100 \mu 1$ were injected slowly at three to four different sites across the tumor to prevent leakage. The resultant local tumor growth was monitored weekly by measuring two perpendicular tumor diameters with a caliper. Tumor volume was calculated by the formula: tumor volume $\left[\mathrm{mm}^{3}\right]=\left[\right.$ length $(\mathrm{mm}) \times(\text { width }(\mathrm{mm})]^{2} \times 0.52$.

Statistical analysis. All quantitative data are reported as means \pm standard deviations (SDs). Statistical analyses were performed with Student's t-test. P-values $<0.05$ were considered significant.

\section{Results}

In vitro characterization of Synco-2D against RCC cells. Because of its twin membrane-fusion mechanisms, Synco-2D 


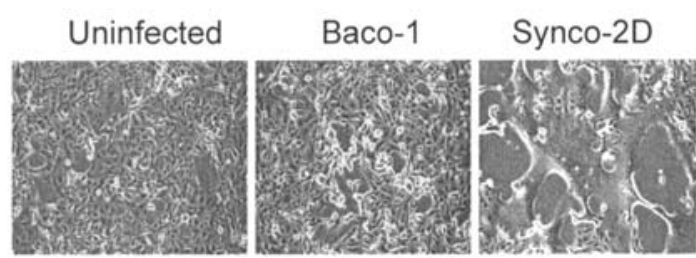

B

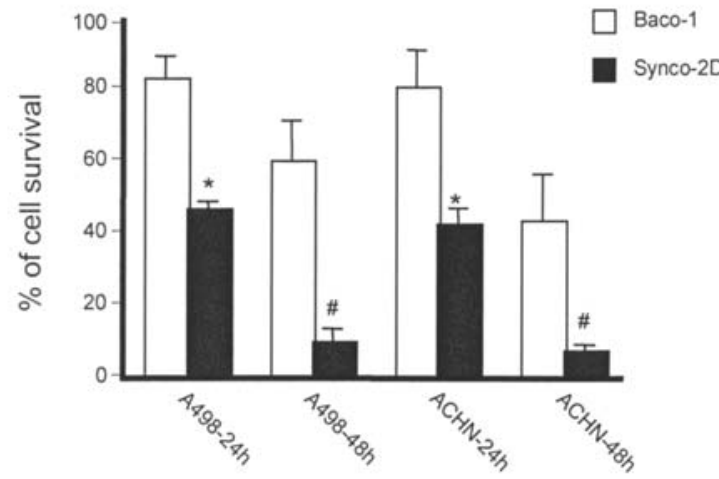

Figure 1. In vitro characterization of Synco-2D in human RCC cells. A, syncytial phenotype. ACHN cell monolayers were either infected with the indicated viruses at $0.01 \mathrm{pfu} / \mathrm{cell}$ or left uninfected. Micrographs were taken at $48 \mathrm{~h}$ after infection (original magnification, $\mathrm{x} 200$ ). B, killing efficiency against human RCC cells. ACHN or A498 cells were seeded into 24-well plates in triplicate and infected with either Baco-1 or Synco-2D at $0.01 \mathrm{pfu} / \mathrm{cell}$, or left uninfected (not shown). Cells were collected at 24 or $48 \mathrm{~h}$ post-infection, and viable cells were counted after trypan-blue staining. Percent cell viability was determined by dividing the number of viable cells in the infected wells by the number of cells in the uninfected well. The data are reported as means \pm SDs. ${ }^{*}$ Significant difference $(\mathrm{P}<0.05$, left panel; $\mathrm{P}<0.01$, right panel) from the corresponding Baco-1 values.

can induce widespread syncytia formation in tumor cells of different tissue origins $(13,20,21)$. To determine if it could also induce cell membrane fusion in RCC, we infected ACHN cells with either Baco-1 or Synco-2D at a low MOI, or left them uninfected. As shown in Fig. 1A, Baco-1 produced plaques that were composed mainly of round cells, typical of HSV infection. Synco-2D, by contrast, produced plaques with a syncytial phenotype, in which the membranes of neighboring cells were fused together, obscuring the usual cell-cell boundaries seen with non-fusogenic HSVs.

Next, we evaluated the in vitro antitumor effects of Synco2D and Baco-1 against two human RCC cell lines, ACHN and A-498. The cells were infected with the viruses at a low dose $(0.01 \mathrm{pfu} / \mathrm{cell})$, so that the ability of virus to infect and to spread within these tumor cells could be examined. The cells were harvested at different times and their viability determined by trypan-blue staining. Regardless of the cell line used, Synco-2D produced superior cytolytic activity (Fig. 1B). Even at this low MOI, it killed nearly $100 \%$ of the tumor cells by $48 \mathrm{~h}$ post-infection, whereas almost half of the tumor cells were still alive in the wells infected with Baco-1.

In vivo antitumor effect of Synco-2D against RCC xenografts. To obtain a valid estimate of the antitumor effect of Synco-2D in vivo, we established RCC xenografts on the right flanks of
A
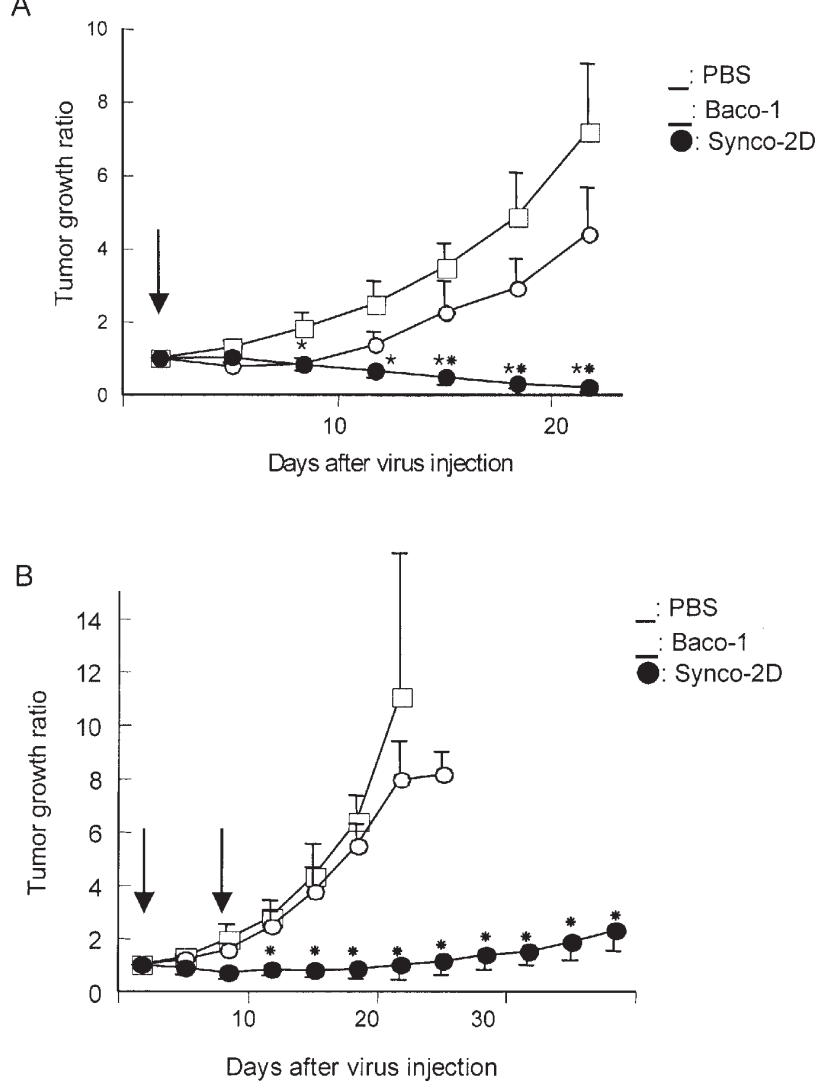

Figure 2. Enhanced oncolytic potency of Synco-2D against human RCC xenografts. A, comparison of oncolytic activity after intratumoral treatment with Synco-2D, Baco-1 or PBS. All tumors received a single injection of virus at $1 \times 10^{7} \mathrm{pfu}$. B, comparison of the oncolytic activity of the same agents delivered intravenously in two doses ( $1 \times 10^{7}$ each, arrows). The tumor growth ratio was determined by dividing the tumor volume measured on the indicated week after virus injection by the tumor volume before treatment. The values are mean \pm SDs ( $n=8$ mice per group). ${ }^{*} \mathrm{P}<0.05$ as compared with $\mathrm{PBS}$; ${ }^{*} \mathrm{P}<0.05$ as compared with both PBS and Baco- 1 .

nude mice by subcutaneous inoculation of $2 \times 10^{5}$ ACHN cells. When tumors reached $\sim 5 \mathrm{~mm}$ in diameter, they were injected directly with a virus dose of $1 \times 10^{7} \mathrm{pfu}$, or the same dose of virus given intratumorally. A repeated intravenous injection with the same virus dose was given 1 week later. Intratumoral injection of Synco-2D produced a striking antitumor effect on RCC xenografts (Fig. 2A). The mean ( \pm SD) tumor growth ratio after Synco-2D treatment decreased to near zero by 3 weeks post-injection, at which time 3 mice in this group were completely tumor-free. Tumors in the remaining 5 mice were significantly smaller than before virotherapy $(\mathrm{P}<0.01)$. Although intratumoral injection of Baco-1 initially shrank tumors, they began to regrow by 12 days post-treatment, attaining a final tumor growth ratio that was 4-fold greater than the pretreatment mean. Systemic delivery of Synco-2D led to initial shrinkage of the tumors with only a gradual increase in the tumor growth ratio over the remaining 20 days of study (Fig. 2B). Baco-1 lacked any demonstrable antitumor effects when administered intravenously.

In vitro characterization of FusOn-H2 against human $\mathrm{RCC}$ cells. Having shown the efficacy of a doubly fusogenic HSV-1based oncolytic virus against RCC, we asked whether an 
A

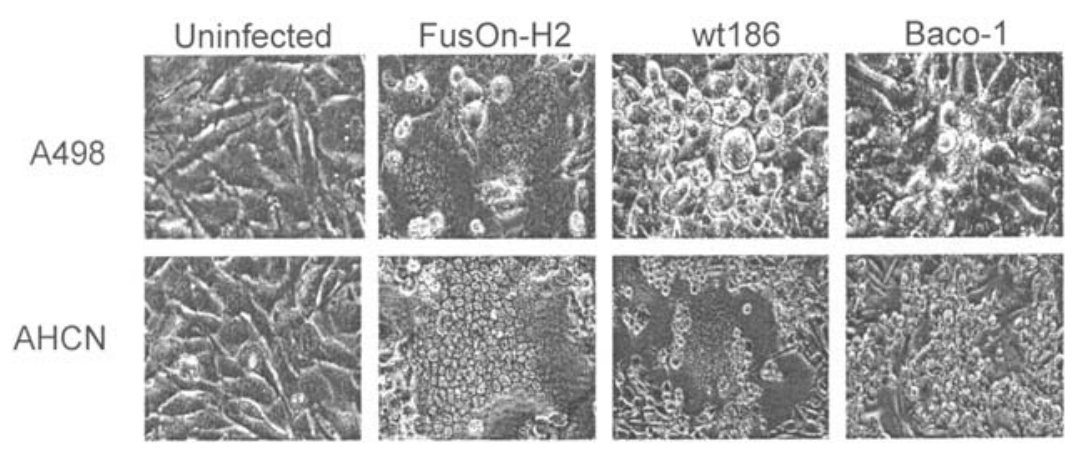

B

$0.1 \mathrm{pfu} / \mathrm{cell}$

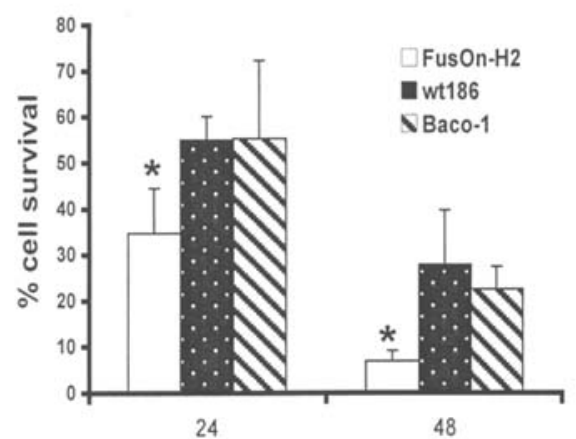

Hours after virus infection
$0.01 \mathrm{pfu} / \mathrm{cell}$

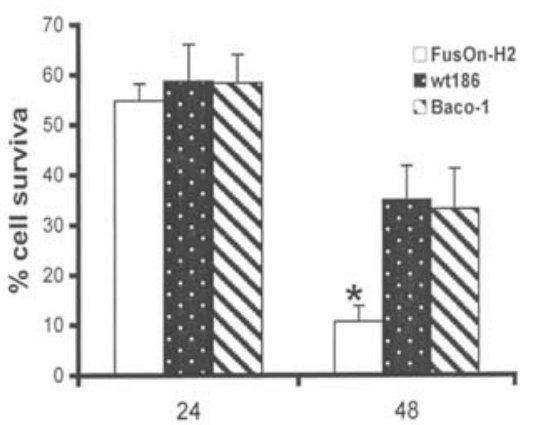

Hours after virus infection

Figure 3. In vitro characterization of FusOn-H2 in human RCC cells. A, syncytial phenotype. A498 or ACHN cell monolayers were infected with the indicated viruses at $0.01 \mathrm{pfu} / \mathrm{cell}$. Micrographs were taken at $48 \mathrm{~h}$ post-infection (original magnification x200). B, in vitro killing efficiency. ACHN cells were seeded into 24-well plates in triplicate and infected with Baco-1, wt186 or FusOn-H2 at 0.1 or 0.01 pfu/cell, or left uninfected (not shown). Cells were collected at 24 or $48 \mathrm{~h}$ post-infection, and viable cells were counted after trypan-blue staining. Percent cell viability was determined by dividing the number of viable cells in the infected wells by the number of cells in the uninfected well. The data are reported as means \pm SDs. * $\mathrm{P}<0.05$ as compared with wt 186 or Baco-1.

oncolytic type $2 \mathrm{HSV}$, FusOn-H2, whose design exploits several unique properties of the virus, might also be useful in RCC treatment. In contrast to HSV-1-based oncolytic viruses (including Baco-1 and Synco-2D) with deletion in the $\gamma 34.5$ gene, FusOn-H2 relies on deletion of the PK domain of the ICP1O gene for its selective replication property (17). To determine the phenotype of FusOn-H2 in human RCC cells, we infected A498 and ACHN cells with this virus, its parental wild-type HSV-2 (strain wt186) and Baco-1. While neither wt186 nor Baco-1 produced syncytia formation, a clearly identifiable syncytial phenotype was evident in cells infected with FusOn-H2 (Fig. 3A), indicating that the fusogenic property of this virus, first noted in tumor cells of breast and ovary origin by $\mathrm{Fu}$ (17), is preserved in RCC cells.

We also compared the in vitro oncolytic effects of FusOn-H2 against one of the RCC lines (ACHN) with those of wt186 and Baco-1, using two different infectious doses (0.1 and $0.01 \mathrm{pfu} / \mathrm{cell})$. While wt186 and Baco- 1 showed similar killing efficiency at each dose and each test interval, FusOn-H2 eradicated significantly more cells than either of these viruses under all conditions except $0.01 \mathrm{pfu} / \mathrm{cell}$ at $24 \mathrm{~h}$ post-infection (Fig. 3B, $\mathrm{P}<0.05$ ).
In vivo oncolytic activity of FusOn-H2 against RCC xenografts. To evaluate the potency of FusOn-H2 against human RCC, we established RCC xenografts through implantation of ACHN cells into the right flanks of nude mice as described earlier. When the tumors attained a diameter of $5 \mathrm{~mm}$, we injected them with either $5 \times 10^{6}$ pfu of FusOn-H2 or PBS (control). The tumors were measured weekly and their growth ratios determined. Although the dose of FusOn-H2 used in this experiment was lower than that of Synco-2D in Fig. 2, the HSV-2-derived virus significantly inhibited tumor growth as early as 1 week post-treatment (Fig. 4A, $\mathrm{P}<0.05$ ). This therapeutic effect was largely maintained throughout the experiment, even though only a single dose of virus was injected.

The long-term therapeutic effect of FusOn-H2 on the RCC xenografts was evaluated by recording animal mortality over 50 days post-implantation. Mice in the PBS-control group began to die or had to be euthanized due to an expanding tumor burden as early as day 24 , and all were dead by day 34 post-implantation (Fig. 4B). By contrast, all mice treated with FusOn-H2 survived for the entire observation period $(\mathrm{P}<0.05)$. 
A

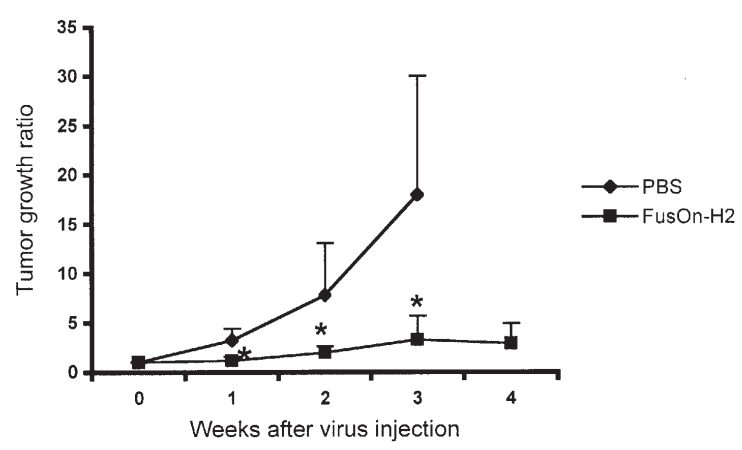

B

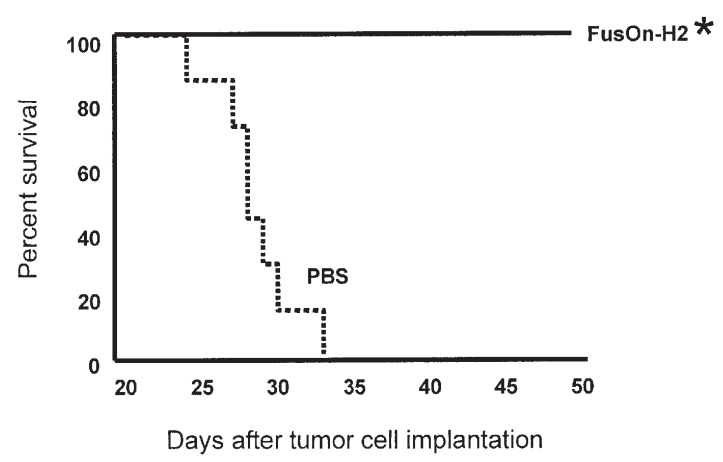

Figure 4. Therapeutic effect of FusOn-H2 against established RCC xenografts. Human RCC xenografts were established on the right flanks of nude mice by subcutaneous inoculation of $2 \times 10^{5} \mathrm{ACHN}$ cells ( $\mathrm{n}=8$ mice per treatment group). When the tumors reached a diameter of approximately $5 \mathrm{~mm}$, they were injected with a single dose of oncolytic HSVs $\left(5 \times 10^{6} \mathrm{pfu}\right)$. A, tumor growth after FusOn-H2 treatment. The tumor growth ratio was determined by dividing the tumor volume measured on the indicated week after virus injection by the tumor volume before treatment. ${ }^{*} \mathrm{P}<0.05$ as compared with the corresponding values for Baco-1. B, Kaplan-Meier plots of survival data collected over the 50 -day observation period. ${ }^{~} \mathrm{P}<0.01$ as compared with PBS.

\section{Discussion}

Developing a safe and effective treatment for advanced RCC remains a daunting challenge. As the kidneys lie deep within the retroperitoneum, RCC may remain clinically silent for most of its course, until the primary tumor progresses to a large size and metastases appear. There are few treatment options for advanced RCC, which is both refractory to cytotoxic chemotherapy and radiotherapy. Surgical resection (radical nephrectomy) with curative intent is the cornerstone of treatment for localized RCC. However, approximately $30 \%$ of patients with RCC present with metastatic disease (1), rendering the benefits of surgery uncertain, and 20-30\% of patients with localized tumors relapse after radical nephrectomy in a median time of 15-18 months (3). The traditional standard first-line treatment for patients with metastatic RCC is IL-2, a biologic response modifier that, in principle, enhances the anticancer activity of terminally differentiated cytolytic T cells. Although the use of highdose IL-2 has been reported to increase objective response rates by approximately $10-20 \%$, with a 1 -year survival rate of approximately $55 \%$ in patients with newly diagnosed metastatic RCC (22), it has not improved long-term survival. Another caveat is that high-dose IL-2 can produce significant toxicity, including capillary leak syndrome, hypotension, severe respiratory distress, and cardiac arrhythmias, all requiring hospitalization for in-patient monitoring (23). Recently, several kinase inhibitors have been approved by FDA for the treatment of metastatic RCC. Among them, it has been shown that, when given as a single therapeutic agent, sunitinab could decrease the size of metastatic RCC by as much as $30 \%(4,24)$. However, such tumor shrinkage occurs in $<30 \%$ of the treated patients.

Despite encouraging results in preclinical studies, the available evidence indicates that oncolytic viruses, although safe, may have only limited antitumor activity on their own. A variety of strategies have been attempted by many different laboratories to enhance the antitumor potency of oncolytic HSVs (25-34). We have chosen to incorporate cell-membrane fusion property into the virus, reasoning that it may not only increase tumor cell killing, but could also facilitate the spread of the oncolytic virus in the tumor tissue. This prediction was substantiated in various solid tumor models $(13,19-21,35)$. Here we demonstrate that the doubly fusogenic Synco-2D virus is superior to a first-generation $\mathrm{HSV}-1$ in the treatment of established RCC. The clear anti-tumor effect of Synco-2D after systemic delivery suggests that this virus might also be useful in therapy for metastatic RCC.

When evaluated by Oyama and colleagues in an RCC xenograft model (12), G207, an HSV-1-based oncolytic virus similar to Baco-1, showed a greater antitumor effect than did Baco-1 in the present study. This discrepancy probably reflects a difference in observation periods: 14 days in the Oyama evaluation versus 4 weeks in ours. It should be noted that Baco-1 and Synco-2D produced similar antitumor effects during the first 2 weeks of follow-up (Fig. 2A); however, with further monitoring, the tumors that were initially shrunk by Baco-1 frequently started to regrow, leading to eventual treatment failure, whereas those treated with Synco-2D continued to shrink and were ultimately eradicated or reduced to an inconsequential size. Responses analogous to these have been observed in other tumor models $(13,20)$.

Intratumor delivery route has been frequently used to evaluate efficacy of virotherapy. However, if virotherapy is ever to succeed as an effective means of treating RCC, it will be critical to demonstrate its efficacy against multifocal disease, which may only be achieved through systemic delivery of the virus. Our results show that intravenously administered Synco-2D, but not the non-fusogenic Baco-1 virus, can inhibit tumor growth, indicating that enhancement strategies such as the twin fusogenic mechanisms of this oncolytic virus are necessary when treating metastatic tumor.

The current generation of oncolytic HSVs were constructed exclusively from HSV-1, yet HSV-2 offers several unique features that make it a very attractive candidate for an oncolytic agent. For example, its genome encodes several protein products that interfere with the host's innate antiviral immunity. One of them, glycoprotein $\mathrm{G}(\mathrm{gG})$ encoded by HSV-2 but not $\mathrm{HSV}-1$, is cleaved post-translationally during processing, resulting in a secreted $\mathrm{N}$-terminal protein of $\sim 300$ amino acids in length and a $\mathrm{C}$-terminal protein that constitutes the cell membrane-anchored gG (36). Recently, it was reported that the secreted form of $\mathrm{gG}$ peptide could actively inhibit natural killer (NK) cell cytotoxicity and accelerate the apoptotic death of NK cell-enriched lymphocytes through its ability to 
recruit and activate phagocytic cells (37). Another example is that the virion host shutoff protein (vhs) encoded by HSV-2 but not HSV-1, interferes with type I interferon production and responsiveness (38). Because innate immunity plays a crucial role in controlling HSV infection (39-41) and strategies to block or modify this arm of the immune system can enhance the antitumor effects of HSV-1-based oncolytic viruses (42), an oncolytic HSV that could evade the host's innate immunity would have an obvious therapeutic advantage over oncolytic viruses derived from HSV-1. With this in mind, we constructed FusOn-H2, which specifically targets tumor cells by exploiting their activated Ras signaling pathway. Our in vitro and in vivo data presented in this article demonstrate that FusOn-H2 has potent oncolytic activity against established RCC xenografts. A single injection of the virus at a very moderate dose led to significant tumor shrinkage in all animals, which translate into their continuous survival over the 50-day observation period. Thus, both Synco-2D and FusOn-H2 display greater antitumor activity than their respective first-generation counterparts while largely retaining their safety features. We believe that the findings reported here warrant further testing of these multifaceted oncolytic viruses in patients with RCC.

\section{References}

1. Jemal A, Murray T, Ward E, et al: Cancer statistics, 2005. CA Cancer J Clin 55: 10-30, 2005.

2. Amato RJ: Chemotherapy for renal cell carcinoma. Semin Oncol 27: 177-186, 2000.

3. Rabinovitch RA, Zelefsky MJ, Gaynor JJ and Fuks Z: Patterns of failure following surgical resection of renal cell carcinoma: implications for adjuvant local and systemic therapy. J Clin Oncol 12: 206-212, 1994.

4. Motzer RJ, Rini BI, Bukowski RM, et al: Sunitinib in patients with metastatic renal cell carcinoma. JAMA 295: 2516-2524, 2006.

5. Martuza RL: Act locally, think globally. Nat Med 3: 1323, 1997.

6. Martuza RL, Malick A, Markert JM, Ruffner KL and Coen DM: Experimental therapy of human glioma by means of a genetically engineered virus mutant. Science 252: 854-856, 1991.

7. Mineta T, Rabkin SD and Martuza RL: Treatment of malignant gliomas using ganciclovir-hypersensitive, ribonucleotide reductase-deficient herpes simplex viral mutant. Cancer Res 54: 3963-3966, 1994.

8. Pyles RB, Warnick RE, Chalk CL, Szanti BE and Parysek LM: A novel multiply-mutated HSV-1 strain for the treatment of human brain tumors. Hum Gene Ther 8: 533-544, 1997.

9. Chou J and Roizman B: The gamma 1(34.5) gene of herpes simplex virus 1 precludes neuroblastoma cells from triggering total shutoff of protein synthesis characteristic of programed cell death in neuronal cells. Proc Natl Acad Sci USA 89: 3266-3270, 1992.

10. Chambers R, Gillespie GY, Soroceanu L, et al: Comparison of genetically engineered herpes simplex viruses for the treatment of brain tumors in a scid mouse model of human malignant glioma. Proc Natl Acad Sci USA 92: 1411-1415, 1995.

11. Mineta T, Rabkin SD, Yazaki T, Hunter WD and Martuza RL: Attenuated multi-mutated herpes simplex virus- 1 for the treatment of malignant gliomas. Nat Med 1: 938-943, 1995.

12. Oyama M, Ohigashi T, Hoshi M, Murai M, Uyemura K and Yazaki T: Treatment of human renal cell carcinoma by a conditionally replicating herpes vector G207. J Urol 165: 1274-1278, 2001

13. Nakamori M, Fu X, Meng F, et al: Effective therapy of metastatic ovarian cancer with an oncolytic herpes simplex virus incorporating two membrane-fusion mechanisms. Clin Cancer Res 9: 2727-2733, 2003.

14. Chung TD, Wymer JP, Smith CC, Kulka M and Aurelian L: Protein kinase activity associated with the large subunit of herpes simplex virus type 2 ribonucleotide reductase (ICP10). J Virol 63: 3389-3398, 1989.
15. Smith CC, Nelson J, Aurelian L, Gober M and Goswami BB: Ras-GAP binding and phosphorylation by herpes simplex virus type 2 RR1 PK (ICP10) and activation of the Ras/MEK/MAPK mitogenic pathway are required for timely onset of virus growth. J Virol 74: 10417-10429, 2000.

16. Farassati F, Yang AD and Lee PW: Oncogenes in Ras signalling pathway dictate host-cell permissiveness to herpes simplex virus 1 . Nat Cell Biol 3: 745-750, 2001.

17. Fu X, Tao L, Cai R, Prigge J and Zhang X: A mutant type 2 herpes simplex virus deleted for the protein kinase domain of the ICP10 gene is a potent oncolytic virus. Mol Ther 13: 882-890, 2006.

18. Milligan GN and Bernstein DI: Generation of humoral immune responses against herpes simplex virus type 2 in the murine female genital tract. Virology 206: 234-241, 1995.

19. Fu X, Tao L, Jin A, Vile R, Brenner M and Zhang X: Expression of a fusogenic membrane glycoprotein by an oncolytic herpes simplex virus provides potent synergistic anti-tumor effect. Mol Ther 7: 748-754, 2003.

20. Nakamori M, Fu X, Pettaway CA and Zhang X: Potent antitumor activity after systemic delivery of a doubly fusogenic oncolytic herpes simplex virus against metastatic prostate cancer. Prostate 60: 53-60, 2004.

21. Nakamori M, Fu X, Rousseau R, Chen SY and Zhang X: Destruction of nonimmunogenic mammary tumor cells by a fusogenic oncolytic herpes simplex virus induces potent antitumor immunity. Mol Ther 9: 658-665, 2004.

22. Fyfe G, Fisher RI, Rosenberg SA, Sznol M, Parkinson DR and Louie AC: Results of treatment of 255 patients with metastatic renal cell carcinoma who received high-dose recombinant interleukin-2 therapy. J Clin Oncol 13: 688-696, 1995.

23. White RL Jr, Schwartzentruber DJ, Guleria A, et al: Cardiopulmonary toxicity of treatment with high dose interleukin-2 in 199 consecutive patients with metastatic melanoma or renal cell carcinoma. Cancer 74: 3212-3222, 1994.

24. Motzer RJ, Michaelson MD, Redman BG, et al: Activity of SU11248, a multitargeted inhibitor of vascular endothelial growth factor receptor and platelet-derived growth factor receptor, in patients with metastatic renal cell carcinoma. J Clin Oncol 24: 16-24, 2006.

25. Advani SJ, Sibley GS, Song PY, et al: Enhancement of replication of genetically engineered herpes simplex viruses by ionizing radiation: a new paradigm for destruction of therapeutically intractable tumors. Gene Ther 5: 160-165, 1998.

26. Chase M, Chung RY and Chiocca EA: An oncolytic viral mutant that delivers the CYP2B1 transgene and augments cyclophosphamide chemotherapy. Nat Biotechnol 16: 444-448, 1998.

27. Spear MA, Sun F, Eling DJ, et al: Cytotoxicity, apoptosis, and viral replication in tumor cells treated with oncolytic ribonucleotide reductase-defective herpes simplex type 1 virus (hrR3) combined with ionizing radiation. Cancer Gene Ther 7: 1051-1059, 2000.

28. Chahlavi A, Todo T, Martuza RL and Rabkin SD: Replicationcompetent herpes simplex virus vector G207 and cisplatin combination therapy for head and neck squamous cell carcinoma. Neoplasia 1: 162-169, 1999.

29. Toyoizumi T, Mick R, Abbas AE, Kang EH, Kaiser LR and Molnar-Kimber KL: Combined therapy with chemotherapeutic agents and herpes simplex virus type 1 ICP34.5 mutant (HSV1716) in human non-small cell lung cancer. Hum Gene Ther 10: 3013-3029, 1999.

30. Andreansky S, He B, van Cott J, et al: Treatment of intracranial gliomas in immunocompetent mice using herpes simplex viruses that express murine interleukins. Gene Ther 5: 121-130, 1998

31. Parker JN, Gillespie GY, Love CE, Randall S, Whitley RJ and Markert JM: Engineered herpes simplex virus expressing IL-12 in the treatment of experimental murine brain tumors. Proc Natl Acad Sci USA 97: 2208-2213, 2000.

32. Todo T, Martuza RL, Dallman MJ and Rabkin SD: In situ expression of soluble B7-1 in the context of oncolytic herpes simplex virus induces potent antitumor immunity. Cancer Res 61: 153-161, 2001.

33. Todo T, Rabkin SD and Martuza RL: Evaluation of ganciclovirmediated enhancement of the antitumoral effect in oncolytic, multimutated herpes simplex virus type 1 (G207) therapy of brain tumors. Cancer Gene Ther 7: 939-946, 2000.

34. Yoon SS, Carroll NM, Chiocca EA and Tanabe KK: Cancer gene therapy using a replication-competent herpes simplex virus type 1 vector. Ann Surg 228: 366-374, 1998. 
35. Fu X and Zhang X: Potent systemic antitumor activity from an oncolytic herpes simplex virus of syncytial phenotype. Cancer Res 62: 2306-2312, 2002.

36. Su HK, Eberle R and Courtney RJ: Processing of the herpes simplex virus type 2 glycoprotein gG-2 results in secretion of a 34,000-Mr cleavage product. J Virol 61: 1735-1737, 1987.

37. Bellner L, Thoren F, Nygren E, Liljeqvist JA, Karlsson A and Eriksson K: A proinflammatory peptide from herpes simplex virus type 2 glycoprotein $\mathrm{G}$ affects neutrophil, monocyte, and NK cell functions. J Immunol 174: 2235-2241, 2005.

38. Duerst RJ and Morrison LA: Herpes simplex virus 2 virion host shutoff protein interferes with type I interferon production and responsiveness. Virology 322: 158-167, 2004.

39. Ashkar AA and Rosenthal KL: Interleukin-15 and natural killer and NKT cells play a critical role in innate protection against genital herpes simplex virus type 2 infection. J Virol 77: 10168-10171, 2003.
40. Grubor-Bauk B, Simmons A, Mayrhofer G and Speck PG: Impaired clearance of herpes simplex virus type 1 from mice lacking CD1d or NKT cells expressing the semivariant V alpha 14-J alpha 281 TCR. J Immunol 170: 1430-1434, 2003.

41. Ahmad A, Sharif-Askari E, Fawaz L and Menezes J: Innate immune response of the human host to exposure with herpes simplex virus type 1: in vitro control of the virus infection by enhanced natural killer activity via interleukin-15 induction. J Virol 74: 7196-7203, 2000.

42. Wakimoto H, Johnson PR, Knipe DM and Chiocca EA: Effects of innate immunity on herpes simplex virus and its ability to kill tumor cells. Gene Ther 10: 983-990, 2003. 